\title{
Solvent Free Amination Reactions of Aryl Bromides at Room Temperature Catalyzed by a ( $\pi$-Allyl)palladium Complex Bearing a Diphosphinidenecyclobutene Ligand
}

\author{
Anil S. Gajare, Kozo Toyota, Masaaki Yoshifuji,* and Fumiyuki Ozawa \\ Department of Chemistry, Graduate School of Science, Tohoku University, Aoba, Sendai \\ 980-8578, Japan and Institute for Chemical Research, Kyoto University, Uji, Kyoto 611- \\ 0011, Japan \\ yoshifj@mail.tains.tohoku.ac.jp
}

\section{Contents of Supporting Information}

(25 Pages)

Page S-1: Title of the Paper, Author's Name and Address along with the Contents.

Page S-2: General Consideration

Page S-3: Characterization Data of Compounds (entries 1-8, Table 2)

Page S-4: Characterization Data of Compounds (entries 9-14, Table 2) and (entries 2 and

3, Table 3)

Page S-5: Characterization Data of Compounds (entries 4-7, Table 3)

Page S-6: Copies of ${ }^{1} \mathrm{H}$ NMR Spectra for Amination Products

Page S-24: A Proposed Catalytic Cycle for Amination Reaction

Page S-25: References 


\section{General Consideration:}

All reactions were performed under an atmosphere of argon in oven-dried glassware. ${ }^{1} \mathrm{H}$ and ${ }^{13} \mathrm{C}$ NMR spectra were recorded at 400 and $100 \mathrm{MHz}$, respectively. Analytical thin layer chromatography (TLC) investigations were performed on a precoated analytical silica-gel aluminum sheet $(0.25 \mathrm{~mm}$ thick). Flash column chromatography separations were performed on spherical neutral silica gel $(70-230$ mesh). The yields reported are isolated yields and are the average of at least two runs. All commercially available reagents were used as received. All reactions were performed without using a dry box and the catalyst $\mathbf{2}$ and $t$-BuOK were weighed in air. 
Diphenylamine $^{1}$ (entry 1, Table 2 and 3, Figure 1): ${ }^{1} \mathrm{H}$ NMR $\left(400 \mathrm{MHz}, \mathrm{CDCl}_{3}\right) \delta$ 7.33-7.29 (td, $J=8.0,2 \mathrm{~Hz}, 4 \mathrm{H}), 7.12(\mathrm{~d}, J=8.0,4 \mathrm{H}), 6.99-6.95(\mathrm{td}, J=8.0,2 \mathrm{~Hz}, 2 \mathrm{H})$, 5.73 (br s, $1 \mathrm{H}) ;{ }^{13} \mathrm{C}$ NMR $\left(100 \mathrm{MHz}, \mathrm{CDCl}_{3}\right) \delta$ 143.6, 129.8, 121.5, 118.3; MS: $m / z$ (rel. int.) $\left(\mathrm{M}^{+}\right) 169(100), 84(9), 77(6), 66(4)$.

Phenethyl(phenyl)amine ${ }^{2}$ (entry 2, Table 2, Figure 2): ${ }^{1} \mathrm{H}$ NMR $\left(400 \mathrm{MHz}, \mathrm{CDCl}_{3}\right) \delta$ $7.40(\mathrm{~m}, 1 \mathrm{H}), 7.28(\mathrm{~m}, 5 \mathrm{H}), 6.81(\mathrm{~m}, 1 \mathrm{H}), 6.70(\mathrm{~d}, 2 \mathrm{H}), 3.75(\mathrm{br} \mathrm{s}, 1 \mathrm{H}), 3.45(\mathrm{dd}, J=6.8$ $\mathrm{Hz}, 7.2 \mathrm{~Hz}, 2 \mathrm{H}), 2.99(\mathrm{dd}, J=6.8 \mathrm{~Hz}, 7.2 \mathrm{~Hz}, 2 \mathrm{H}) ;{ }^{13} \mathrm{C} \mathrm{NMR}\left(100 \mathrm{MHz}, \mathrm{CDCl}_{3}\right) \delta 148.5$, 139.8, 129.7, 129.3, 129.1, 126.9, 117.9, 113.5, 45.5, 36.0; MS: $m / z$ (rel. int.) $\left(\mathrm{M}^{+}\right) 197$ (9), 183 (3), 169 (1), 106 (100), 91 (6), 77 (12), 51 (3).

Benzyl-p-tolylamine ${ }^{1}$ (entry 3, Table 2, Figure 3): ${ }^{1} \mathrm{H}$ NMR $\left(400 \mathrm{MHz}, \mathrm{CDCl}_{3}\right) \delta$ 7.17-7.30 (m, 5H), 6.73-6.77 (d, $J=7.2 \mathrm{~Hz}, 2 \mathrm{H}), 6.68-6.66$ (d $J=7.6 \mathrm{~Hz}, 2 \mathrm{H}), 4.32$ (s, $2 \mathrm{H}) 4.0(\mathrm{br} \mathrm{s}, 1 \mathrm{H}), 2.38(\mathrm{~s}, 3 \mathrm{H}) ;{ }^{13} \mathrm{C}$ NMR $\left(100 \mathrm{MHz}, \mathrm{CDCl}_{3}\right) \delta$ 148.7, 136.8, 130.3, 129.8, 129.7, 127.9, 113.3, 48.6, 21.8; MS: $m / z$ (rel. int.) $\left(\mathrm{M}^{+}\right) 197$ (63), 105 (100), 97 (3), 77 (13), 51 (3).

(Naphthalen-1-ylmethyl)phenylamine ${ }^{3}$ (entry 4, Table 2, Figure 4): ${ }^{1} \mathrm{H}$ NMR (400 $\left.\mathrm{MHz}, \mathrm{CDCl}_{3}\right) \delta 8.10(\mathrm{~d}, J=8.8 \mathrm{~Hz}, 1 \mathrm{H}), 7.84(\mathrm{~m}, 2 \mathrm{H}), 7.45(\mathrm{~m}, 4 \mathrm{H}), 7.23(\mathrm{~m}, 2 \mathrm{H}), 6.72$ $(\mathrm{m}, 3 \mathrm{H}), 4.78(\mathrm{~s}, 2 \mathrm{H}), 4.04(\mathrm{br} \mathrm{s}, 1 \mathrm{H}),{ }^{13} \mathrm{C}$ NMR $\left(100 \mathrm{MHz}, \mathrm{CDCl}_{3}\right) \delta \quad 148.7,134.8$, 134.4, 132.0, 129.8, 129.2, 128.7, 126.8, 126.5, 126.3, 126.0, 124.0, 118.1, 113.2, 46.8; MS: $m / z$ (rel. int.) $\left(\mathrm{M}^{+}\right) 233$ (47), 141 (100), 115 (11), 77 (1).

Phenyl(1-phenylethyl)amine ${ }^{4}$ (entry 5, Table 2, Figure 5): ${ }^{1} \mathrm{H}$ NMR $(400 \mathrm{MHz}$, $\left.\mathrm{CDCl}_{3}\right) \delta$ 7.38-7.30 (m, 4H), 7.08-6.95 (m, 5H) 5.69 (brs, $\left.1 \mathrm{H}\right) ;{ }^{13} \mathrm{C}$ NMR (100 MHz, $\left.\mathrm{CDCl}_{3}\right) \delta 142.6,142.5,132.3,129.9,121.8,119.2,118.4,112.7$; MS: $\mathrm{m} / z$ (rel. int.) $\left(\mathrm{M}^{+}\right)$ 249 (97), 248 (15), 247 (100), 167 (46), 141 (4), 83 (13), 76 (5), 65 (4), 51 (4).

Hexadecyl(phenyl)amine ${ }^{5}$ (entry 6, Table 2, Figure 6): ${ }^{1} \mathrm{H}$ NMR (400 $\left.\mathrm{MHz}, \mathrm{CDCl}_{3}\right) \delta$ $719(\mathrm{~d}, 2 \mathrm{H}), 6.70(\mathrm{t}, J=7.2 \mathrm{~Hz}, 1 \mathrm{H}), 6.63(\mathrm{~d}, 2 \mathrm{H}), 3.61$ (brs, $1 \mathrm{H}), 3.12(\mathrm{t}, 2 \mathrm{H}), 1.61(\mathrm{~m}$, $2 \mathrm{H}), 1.30(\mathrm{~m}, 26 \mathrm{H}), 0.90(\mathrm{t}, 3 \mathrm{H}) ;{ }^{13} \mathrm{C}$ NMR $\left(100 \mathrm{MHz}, \mathrm{CDCl}_{3}\right) \delta 148.9,129.6,117.5$, 113.1, 44.4, 14.5; MS: $m / z$ (rel. int.) (M $\left.\mathrm{M}^{+}\right) 317$ (57), 106 (44), 77 (1), 55 (2).

(4-Methoxyphenyl)phenylamine ${ }^{1}$ (entry 7 and 8, Table 2, Figure 7): ${ }^{1} \mathrm{H}$ NMR (400 $\left.\mathrm{MHz}, \mathrm{CDCl}_{3}\right) \delta 7.24(\mathrm{~d}, J=7.5 \mathrm{~Hz}, 2 \mathrm{H}), 7.11(\mathrm{~d}, J=7.5 \mathrm{~Hz}, 2 \mathrm{H}), 6.87(\mathrm{~m}, 5 \mathrm{H}), 5.51(\mathrm{~s}$, $1 \mathrm{H}), 3.84(\mathrm{~s}, 3 \mathrm{H}) ;{ }^{13} \mathrm{C} \mathrm{NMR}\left(100 \mathrm{MHz}, \mathrm{CDCl}_{3}\right) \delta$ 155.8, 145.7, 136.6, 129.7, 122.6, 120.1, 116.1, 115.1, 56.0; MS: $m / z$ (rel. int.) (M $\left.\mathrm{M}^{+}\right)$199(96), 184 (100), 167 (5), 154 (6), 129 (6), 99 (4), 77 (8), 51 (4). 
Ethyl 4-Phenylaminobenzoate ${ }^{6}$ (entry 9, Table 2, Figure 8): ${ }^{1} \mathrm{H}$ NMR (400 MHz, $\left.\mathrm{CDCl}_{3}\right) \delta 7.93-7.97(\mathrm{dd}, J=2.4 \mathrm{~Hz}, 8.8 \mathrm{~Hz} 2 \mathrm{H}), 7.18-7.38(\mathrm{~m}, 4 \mathrm{H}), 7.03-7.11(\mathrm{~m}, 3 \mathrm{H})$, 6.06 (brs, 1H), $4.34(\mathrm{q}, J=7.1 \mathrm{~Hz}, 2 \mathrm{H}) 1.38(\mathrm{t}, J=7.1 \mathrm{~Hz}, 3 \mathrm{H}) ;{ }^{13} \mathrm{C}$ NMR $(100 \mathrm{MHz}$, $\left.\mathrm{CDCl}_{3}\right) \delta 166.9,148.3,141.3,131.8,129.9,122.8,120.7,115.0,60.8,14.8 ; \mathrm{MS}: \mathrm{m} / z$ (rel. int.) $\left(\mathrm{M}^{+}\right) 241$ (100), 213 (21), 196 (55), 167 (23), 98 (6), 83 (8), 77 (3).

(2-Methoxyphenyl)phenylamine ${ }^{7}$ (entry 10, Table 2, Figure 9): ${ }^{1} \mathrm{H}$ NMR $(400 \mathrm{MHz}$, $\left.\mathrm{CDCl}_{3}\right) \delta$ 7.29-7.35 (m, 3H), 7.17-7.19 (m, 2H), 6.99 (t, J=7.2 Hz, 1H), 6.89-6.94 (m, 3H) 6.18 (br s, $1 \mathrm{H}), 3.91(\mathrm{~s}, 3 \mathrm{H}) ;{ }^{13} \mathrm{C} \mathrm{NMR}\left(100 \mathrm{MHz}, \mathrm{CDCl}_{3}\right) \delta 148.8,143.2,133.5$, 129.7, 121.6, 121.3, 120.4, 119.1, 115.2, 111.0, 56.1; MS: $m / z$ (rel. int.) (M $\left.{ }^{+}\right) 199(100)$, 184 (62), 167 (7), 156 (14), 129 (9), 105 (10), 100 (4), 77 (51), 51 (13).

(4-t-Butylphenyl)phenylamine ${ }^{8}$ (entry 11, Table 2, Figure 10): ${ }^{1} \mathrm{H}$ NMR $(400 \mathrm{MHz}$, $\left.\mathrm{CDCl}_{3}\right) \delta 7.27(\mathrm{~m}, 4 \mathrm{H}), 6.90(\mathrm{~m}, 5 \mathrm{H}), 5.66$ (brs, $\left.1 \mathrm{H}\right), 1.34(\mathrm{~s}, 9 \mathrm{H}) ;{ }^{13} \mathrm{C} \mathrm{NMR}(100 \mathrm{MHz}$, $\left.\mathrm{CDCl}_{3}\right) \delta 144.1,140.7,129.7,126.6,120.8,118.5,117.7,117.5,34.6,31.7$; MS: $\mathrm{m} / z$ (rel. int.) $\left(\mathrm{M}^{+}\right) 225$ (58), 210 (100), 195 (6), 182 (9), 168 (5), 105 (6), 91 (8),77 (13).

(3,5-Dimethylphenyl)phenylamine ${ }^{9}$ (entry 12, Table 2, Figure 11): ${ }^{1} \mathrm{H}$ NMR (400 $\left.\mathrm{MHz} \mathrm{CDCl}_{3}\right) \delta$ 7.07-7.31 (m, 5H), $6.91(\mathrm{~s}, 1 \mathrm{H}) 6.61(\mathrm{~s}, 1 \mathrm{H}), 5.64$ (brs, 1H), $2.29(\mathrm{~s}, 6 \mathrm{H})$; ${ }^{13} \mathrm{C}$ NMR $\left(100 \mathrm{MHz}, \mathrm{CDCl}_{3}\right) \delta 143.8,143.5,139.5,129.7,123.3,121.2,118.3,116.0$, 21.9; MS: $m / z$ (rel. int.) $\left(\mathrm{M}^{+}\right) 197$ (100), 181 (13), 167 (5), 90 (4), 77 (13), 51 (2).

(4-Bromophenyl)phenylamine ${ }^{4}$ (entry 13 and 14 Table 2, Figure 12): ${ }^{1} \mathrm{H}$ NMR (400 $\left.\mathrm{MHz}, \mathrm{CDCl}_{3}\right) \delta 7.41-7.24(\mathrm{~m}, 5 \mathrm{H}), 7.10(\mathrm{t}, J=8.0 \mathrm{~Hz}, 2 \mathrm{H}) 6.65(\mathrm{t}, J=8.0 \mathrm{~Hz}, 1 \mathrm{H}), 6.53$ $(\mathrm{d}, J=8.0 \mathrm{~Hz}, 2 \mathrm{H}), 4.49$ (q, $J=6.8 \mathrm{~Hz}, 1 \mathrm{H}), 4.05$ (brs, $1 \mathrm{H}), 1.54(\mathrm{~d}, J=6.8 \mathrm{~Hz}, 3 \mathrm{H}) ;{ }^{13} \mathrm{C}$ NMR $\left(100 \mathrm{MHz}, \mathrm{CDCl}_{3}\right) \delta 147.7,145.7,129.5,129.1,127.3,126.3,117.7,113.7,53.8$, 25.47; MS: $m / z$ (rel. int.) $\left(\mathrm{M}^{+}\right) 197$ (81), 182 (100), 120 (9), 105 (79), 99 (4), 93 (52), 77 (28), 65 (4), 51 (8).

$\boldsymbol{N}$-Methyldiphenylamine ${ }^{10}$ (entry 2, Table 3, Figure 13): ${ }^{1} \mathrm{H}$ NMR (400 $\left.\mathrm{MHz}, \mathrm{CDCl}_{3}\right) \delta$ 7.46-7.44 (m, 4H), 7.29-7.15 (m, 6H), $\left.3.49(\mathrm{~s}, 3 \mathrm{H}) ;{ }^{13} \mathrm{C} \mathrm{NMR} \mathrm{(100} \mathrm{MHz,} \mathrm{CDCl}_{3}\right) \delta$ 149.6, 129.8, 121.9, 121.0, 40.8; MS: $m / z$ (rel. int.) $\left(\mathrm{M}^{+}\right) 183$ (100), 167 (17), 104 (7), 83 (3) 77 (14), 51 (6).

Benzhydrylidene(phenyl)amine ${ }^{11}$ (entry 3, Table 3, Figure 14): ${ }^{1} \mathrm{H}$ NMR (400 MHz, $\left.\mathrm{CDCl}_{3}\right) \delta 7.82(\mathrm{~m}, 2 \mathrm{H}), 7.59-7.78(\mathrm{~m}, 3 \mathrm{H}), 7.47-7.53(\mathrm{~m}, 4 \mathrm{H}), 7.12-7.40(\mathrm{~m}, 5 \mathrm{H})$, 6.73-6.75 (m, 1H); ${ }^{13} \mathrm{C}$ NMR (100 MHz, $\left.\mathrm{CDCl}_{3}\right) \delta$ 168.7, 157.6, 132.8, 131.1, 130.5, $129.9,129.7,128.9,128.7,128.6,128.3,123.5,121.4$; MS: $m / z$ (rel. int.) $\left(\mathrm{M}^{+}\right) 257$ (100), $180(80), 165(34), 76(41), 51$ (10). 
4-Phenylmorpholine ${ }^{1}$ (entry 4, Table 3, Figure 15): ${ }^{1} \mathrm{H}$ NMR $\left(400 \mathrm{MHz}, \mathrm{CDCl}_{3}\right) \delta$ 7.34-7.38 (m, 2H), 6.95-6.99 (m, 3H), 3.91-3.93 (t, 4H), 3.19-3.22 (t, 4H); ${ }^{13} \mathrm{C}$ NMR $\left(100 \mathrm{MHz}, \mathrm{CDCl}_{3}\right) \delta 151.8,129.7,120.5,116.2,67.4,49.8$; MS: $m / z$ (rel. int.) $\left(\mathrm{M}^{+}\right)$ 163(100), 132 (5), 105 (86), 91 (3) 77 (23), 51 (8).

1-Phenylpiperidine ${ }^{1}$ (entry 5, Table 3, Figure 16): ${ }^{1} \mathrm{H}$ NMR (400 $\left.\mathrm{MHz}, \mathrm{CDCl}_{3}\right) \delta$ 7.27-7.31 (m, 3H), 6.85-7.0 (m, 2H), $6.85(\mathrm{t}, 1 \mathrm{H}), 3.19(\mathrm{t}, 4 \mathrm{H}), 1.64-1.79(\mathrm{~m}, 4 \mathrm{H})$, $1.59-1.63(\mathrm{~m}, 2 \mathrm{H}),{ }^{13} \mathrm{C} \mathrm{NMR}\left(100 \mathrm{MHz}, \mathrm{CDCl}_{3}\right) \delta$ 152.7, 129.5, 119.6, 117.0, 51.1, 29.3, 26.4; MS: $m / z$ (rel. int.) ( $\left.\mathrm{M}^{+}\right) 161$ (83), 160 (100), 146 (3), 132 (7), 120 (12), 105 (22), 91 (6), 77 (15), $51(5)$.

1-(4-t-Butylphenyl)piperidine ${ }^{12}$ (entry 6, Table 3, Figure 17): ${ }^{1} \mathrm{H}$ NMR $(400 \mathrm{MHz}$, $\left.\mathrm{CDCl}_{3}\right) \delta 7.28(\mathrm{~d}, J=8.8 \mathrm{~Hz}, 2 \mathrm{H}), 6.90(\mathrm{~d}, J=8.8 \mathrm{~Hz}, 2 \mathrm{H}), 3.13$ (t, d, $\left.J=5.5 \mathrm{~Hz}, 4 \mathrm{H}\right)$, $1.70-1.76(\mathrm{~m}, 4 \mathrm{H}), 1.58-1.61(\mathrm{~m}, 2 \mathrm{H}), 1.31(\mathrm{~s}, 9 \mathrm{H}) ;{ }^{13} \mathrm{C} \mathrm{NMR}\left(100 \mathrm{MHz}, \mathrm{CDCl}_{3}\right) \delta$ 150.4, 142.3, 126.1, 116.6, 51.3, 34.3, 31.9, 26.4, 24.8; MS: $m / z$ (rel. int.) $\left(\mathrm{M}^{+}\right) 217$ (35), $202(100)$.

2-Phenyl-1,2,3,4-tetrahydroisoquinoline ${ }^{4}$ (entry 7, Table 3, Figure 18): ${ }^{1} \mathrm{H}$ NMR (400 $\left.\mathrm{MHz}, \mathrm{CDCl}_{3}\right) \delta 7.40(\mathrm{~m}, 2 \mathrm{H}), 7.27(\mathrm{~m}, 4 \mathrm{H}), 7.08(\mathrm{~d}, 2 \mathrm{H}), 6.93(\mathrm{t}, J=7.0 \mathrm{~Hz}, 1 \mathrm{H}), 4.51$ (s, 2H), 3.65 (t, $J=5.0 \mathrm{~Hz}, 2 \mathrm{H}), 3.07(\mathrm{t} J=5.0 \mathrm{~Hz}, 2 \mathrm{H}) ;{ }^{13} \mathrm{C} \mathrm{NMR}\left(100 \mathrm{MHz}, \mathrm{CDCl}_{3}\right)$ $\delta$ 151.1, 135.4 134.9, 129.7, 129.0, 127.0, 126.8, 126.5, 119.1, 115.6, 51.2, 47.0, 29.6; MS: $m / z$ (rel. int.) (M+) 209 (97), 208 (100), 181 (5), 115 (4), 105 (58), 77 (13), 51 (4). 

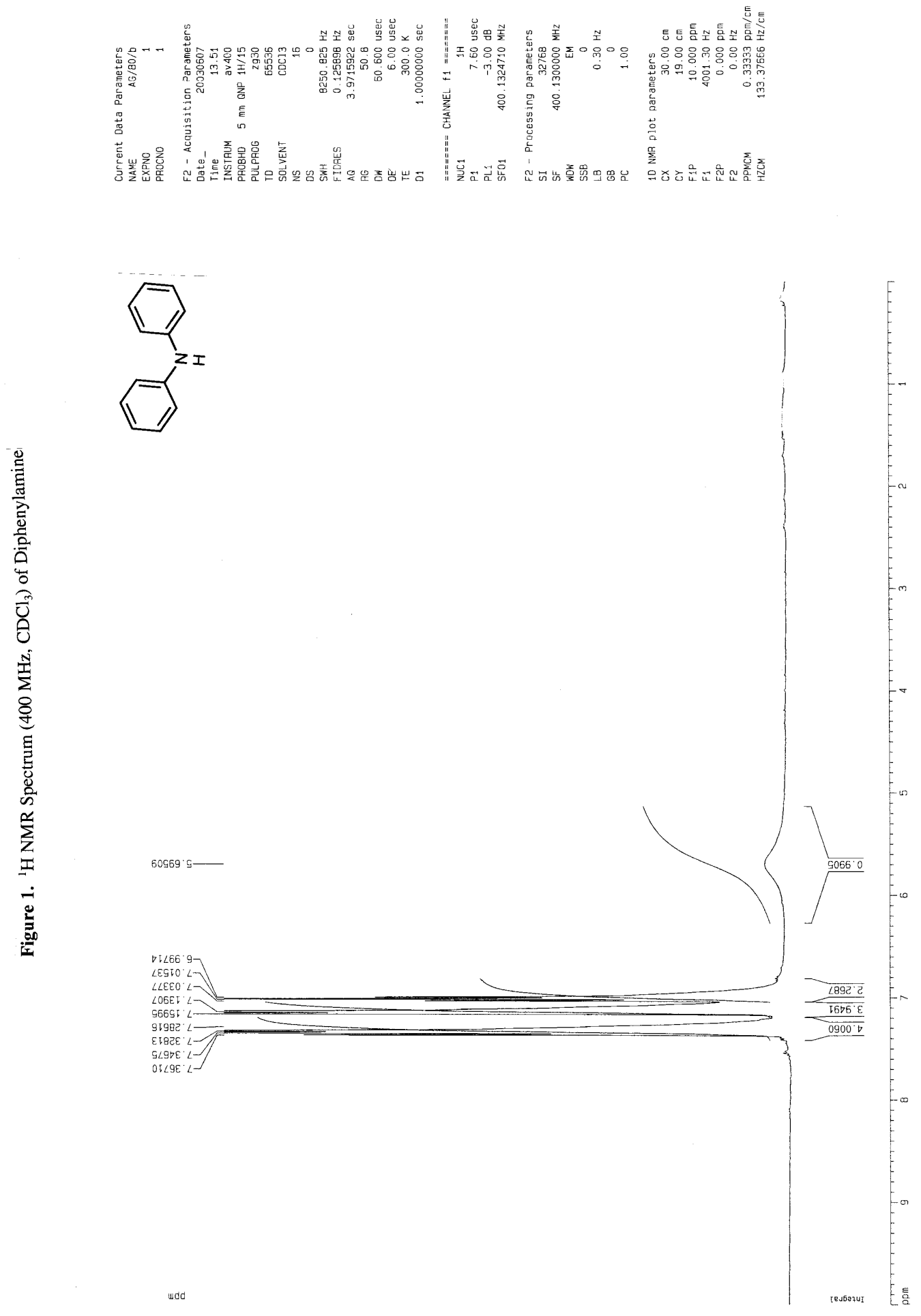

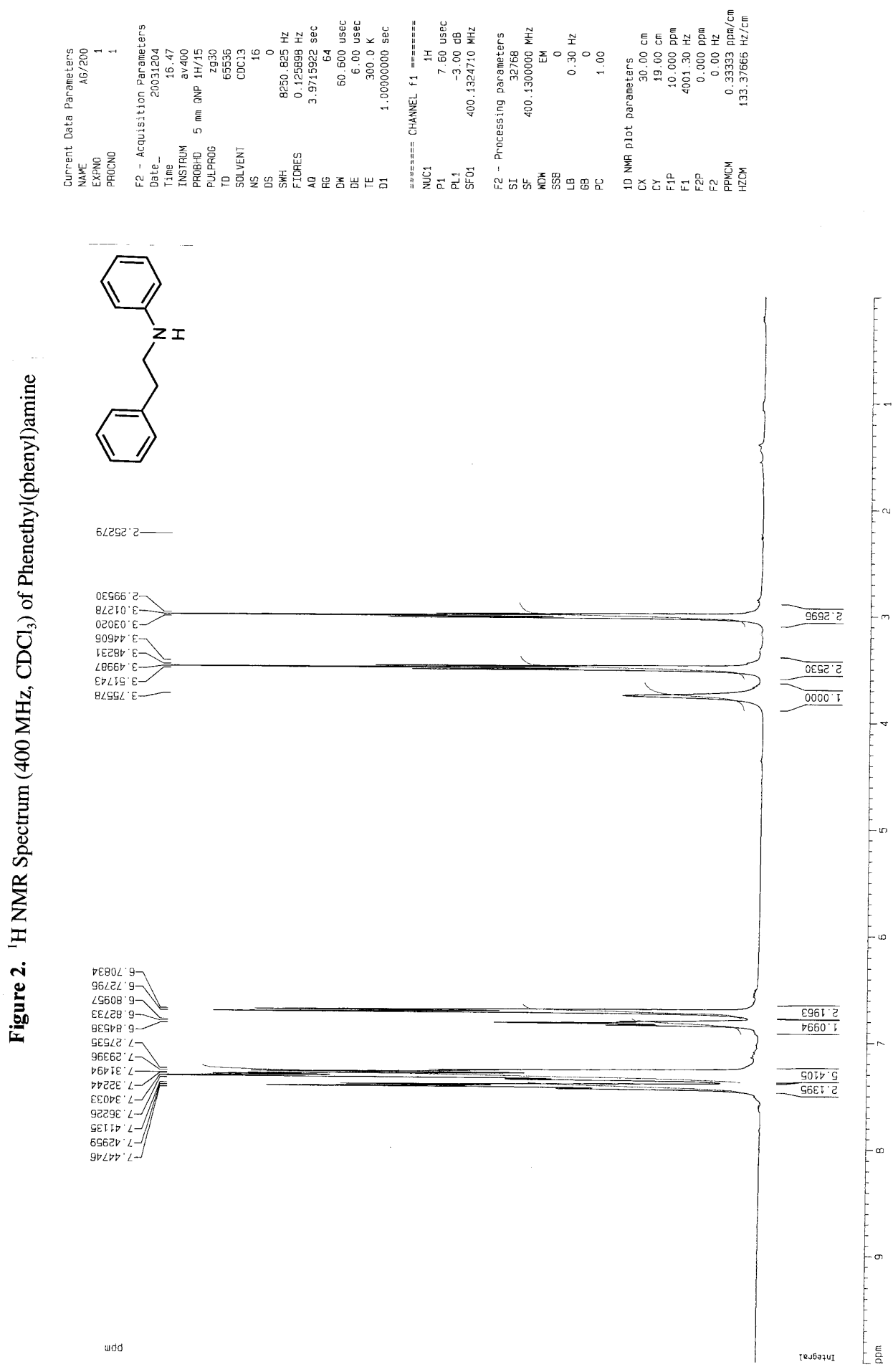

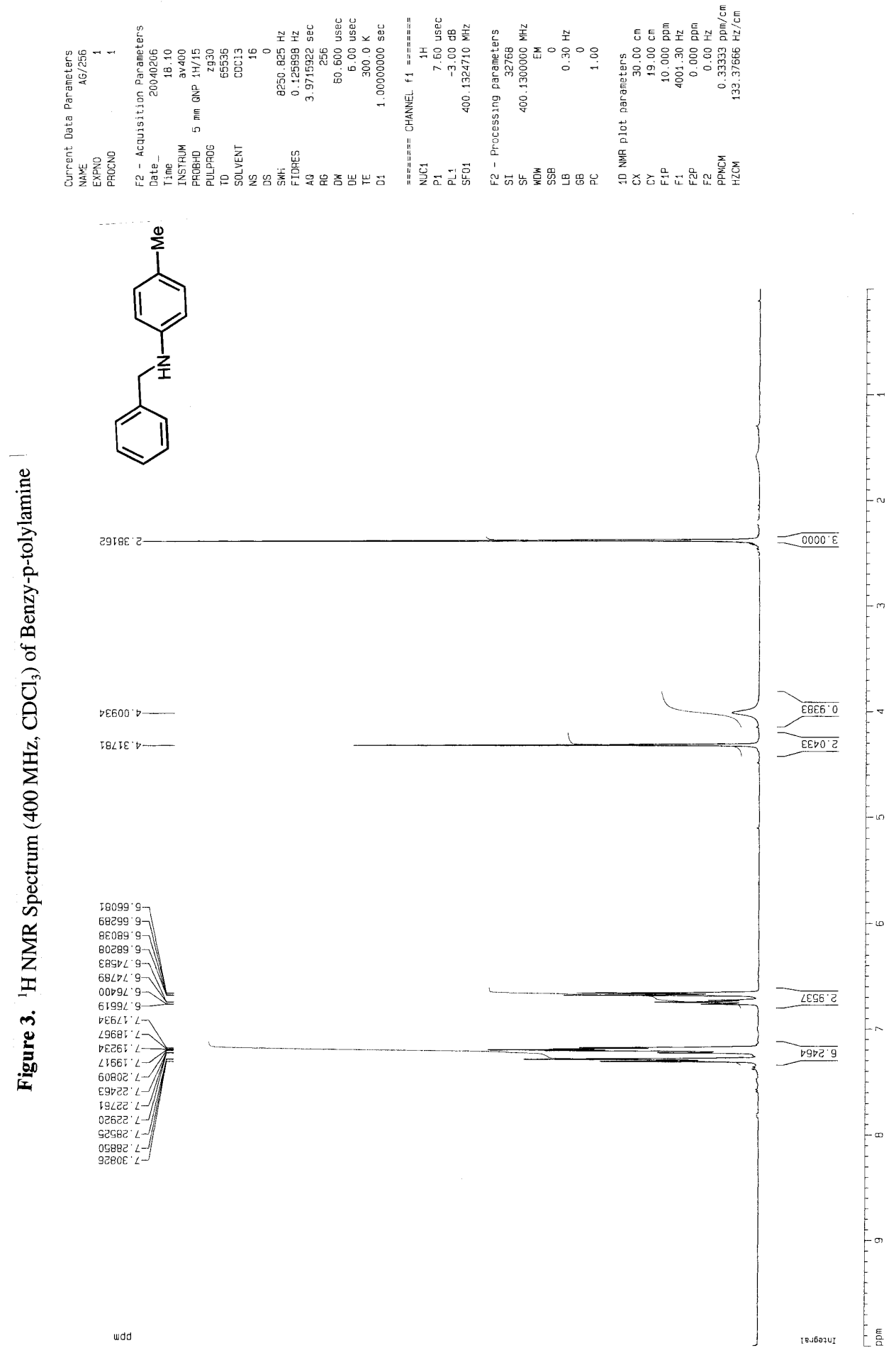

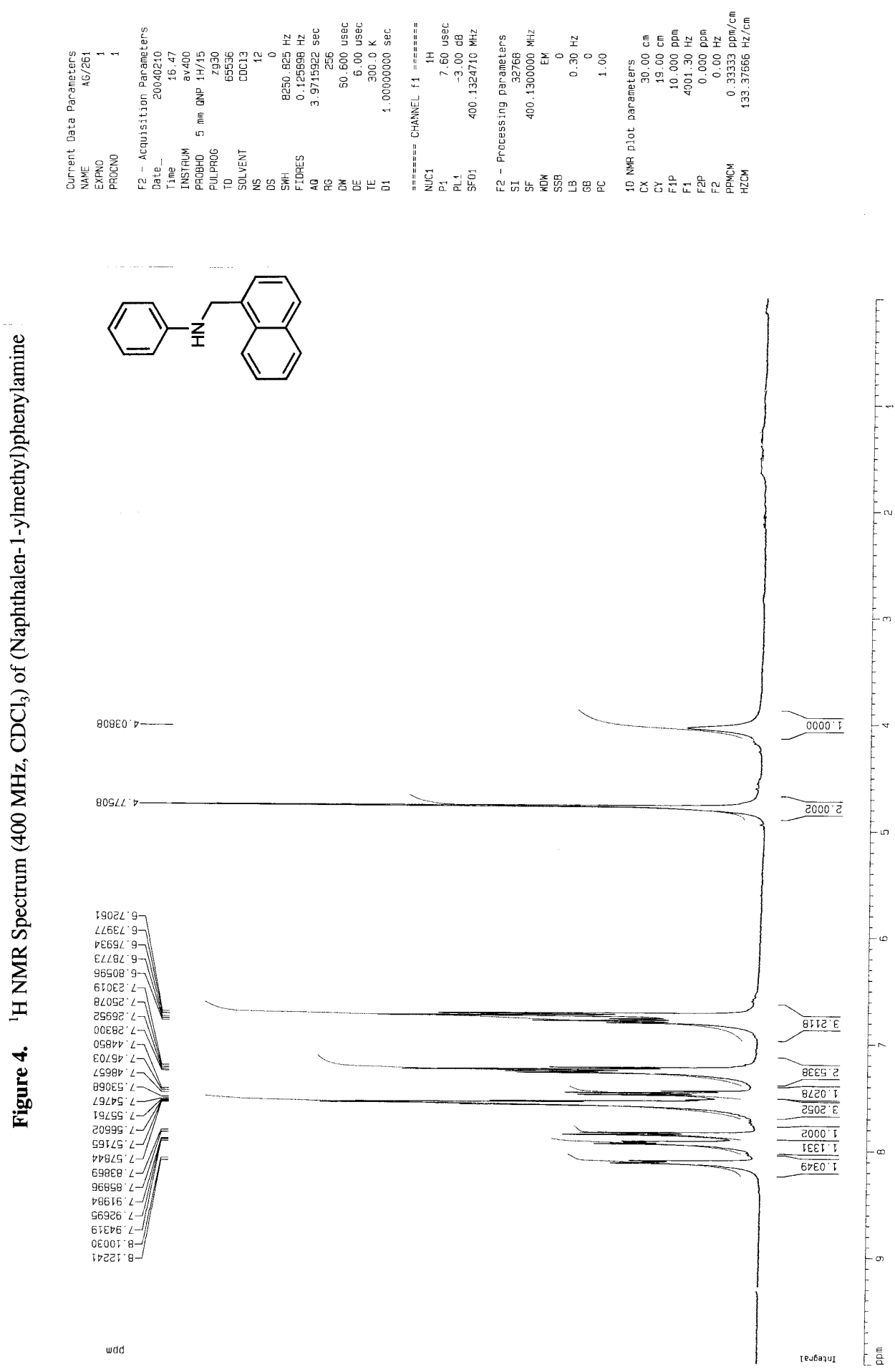


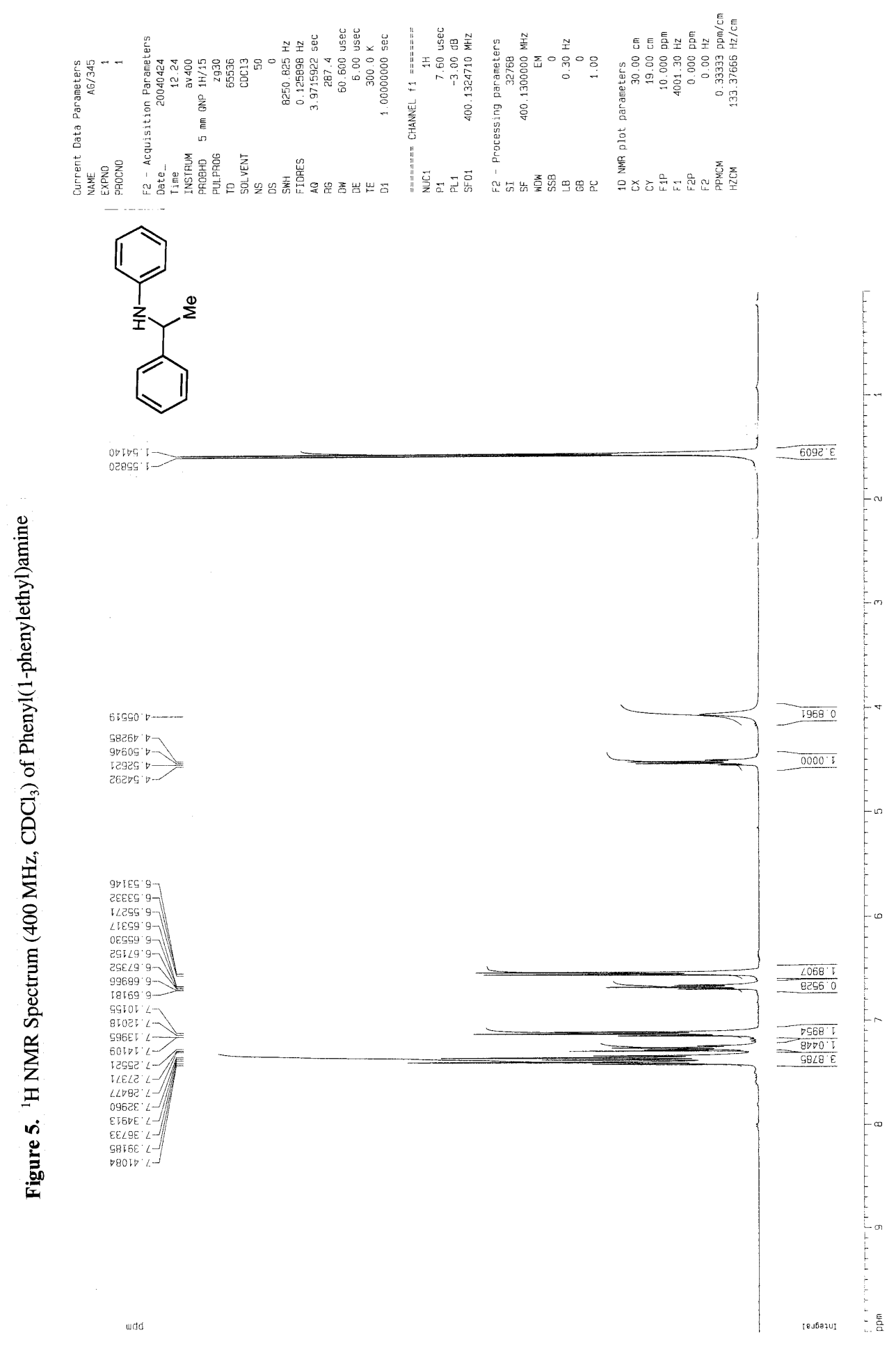



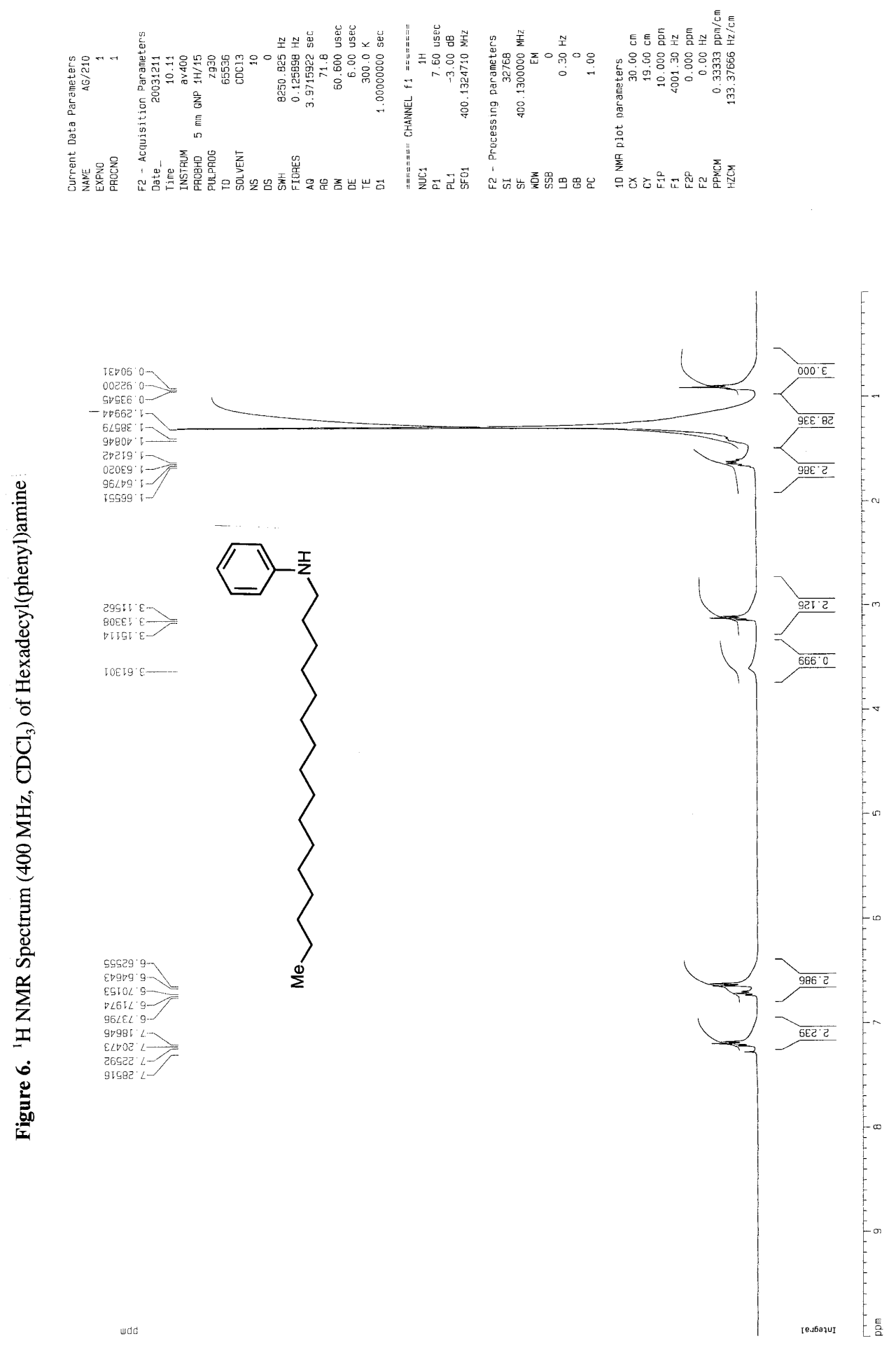

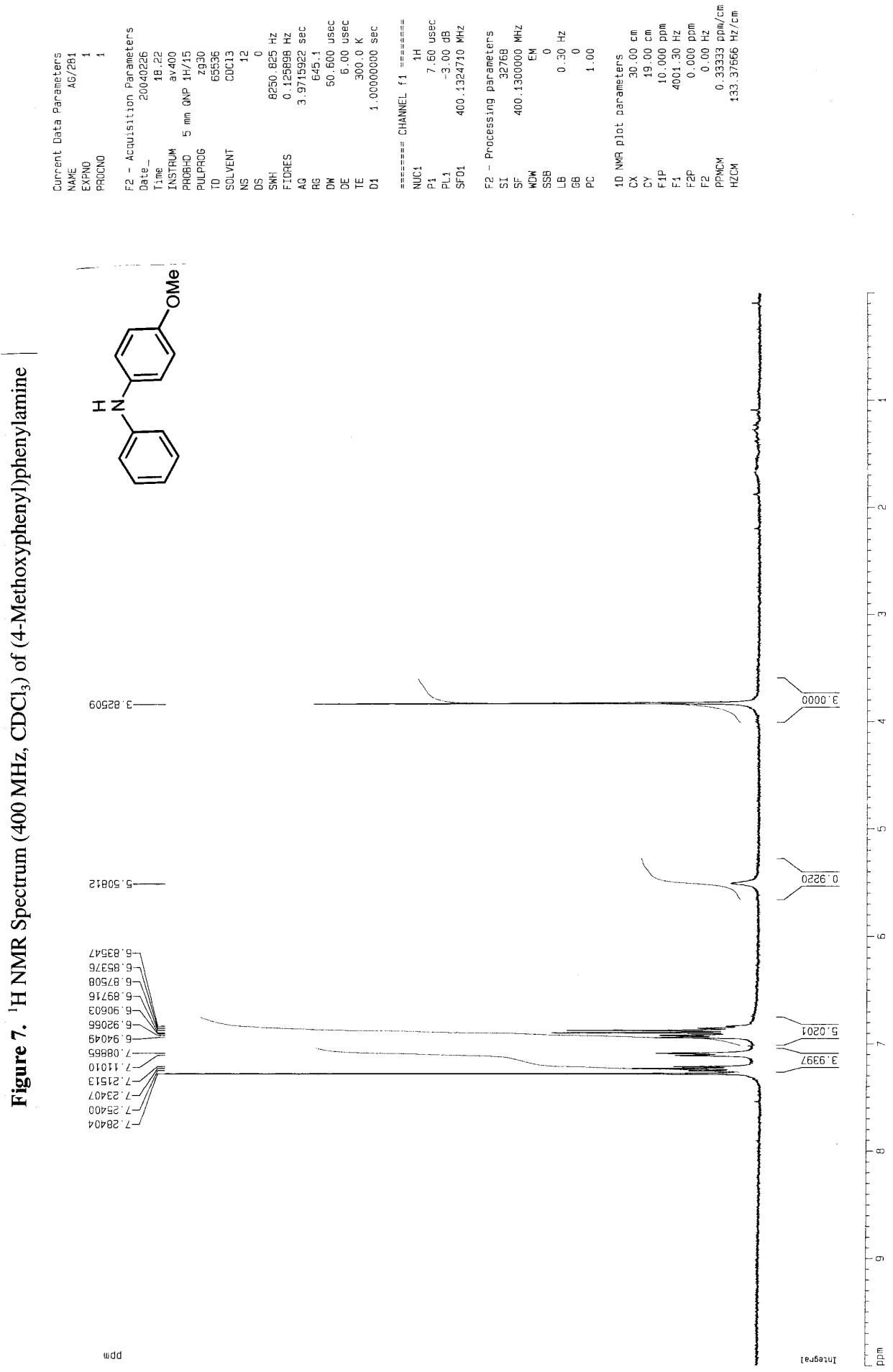

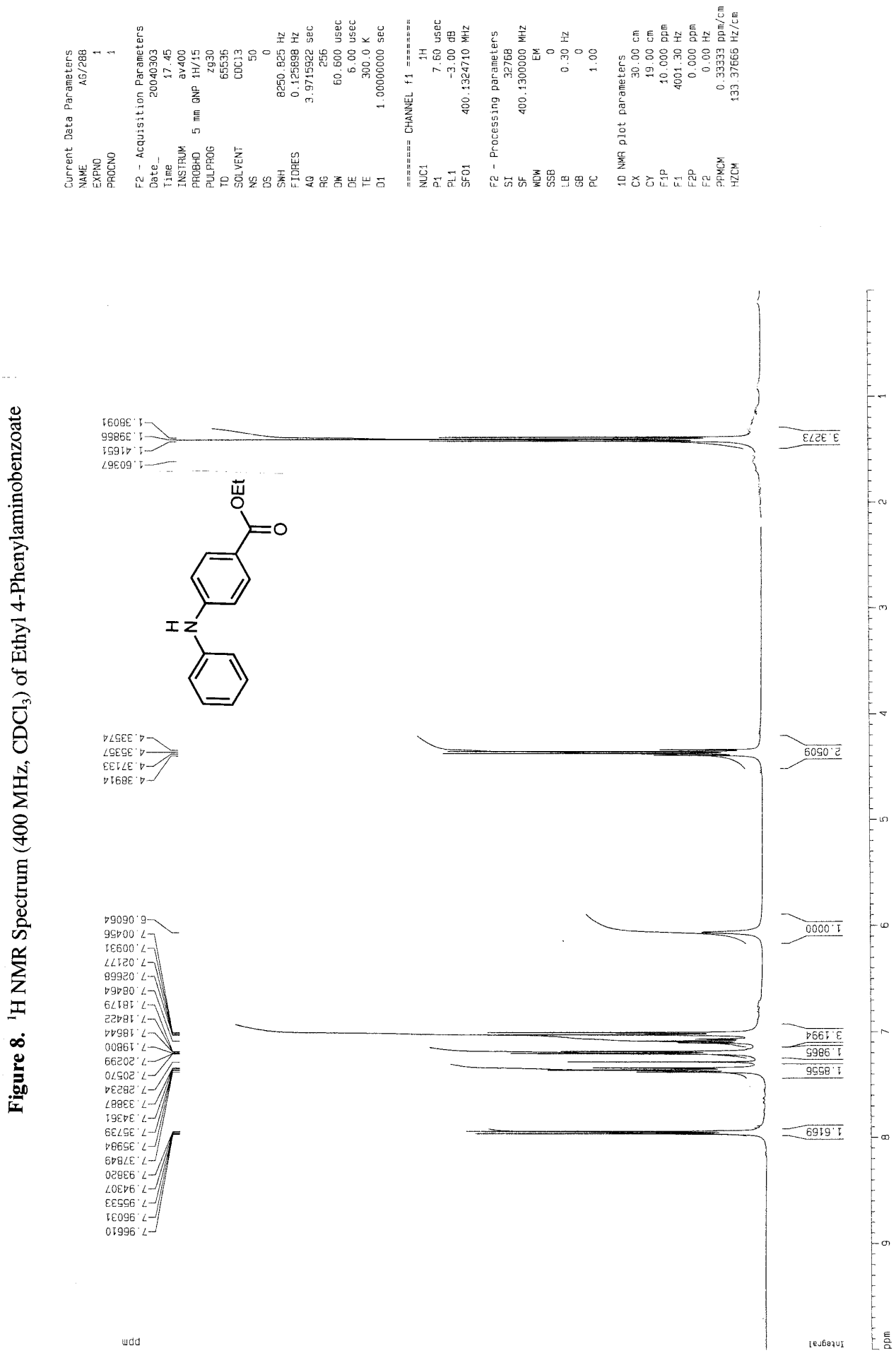

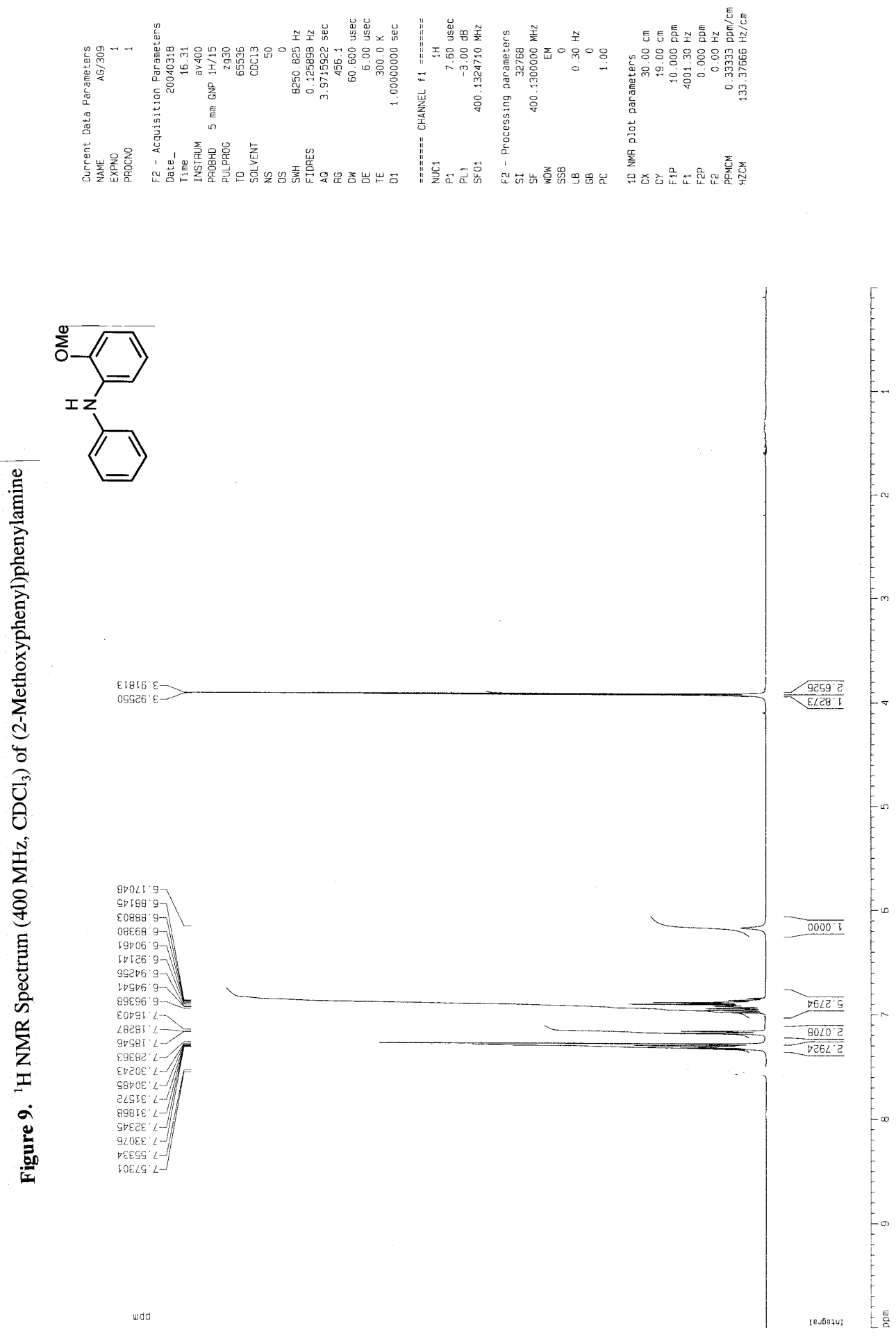

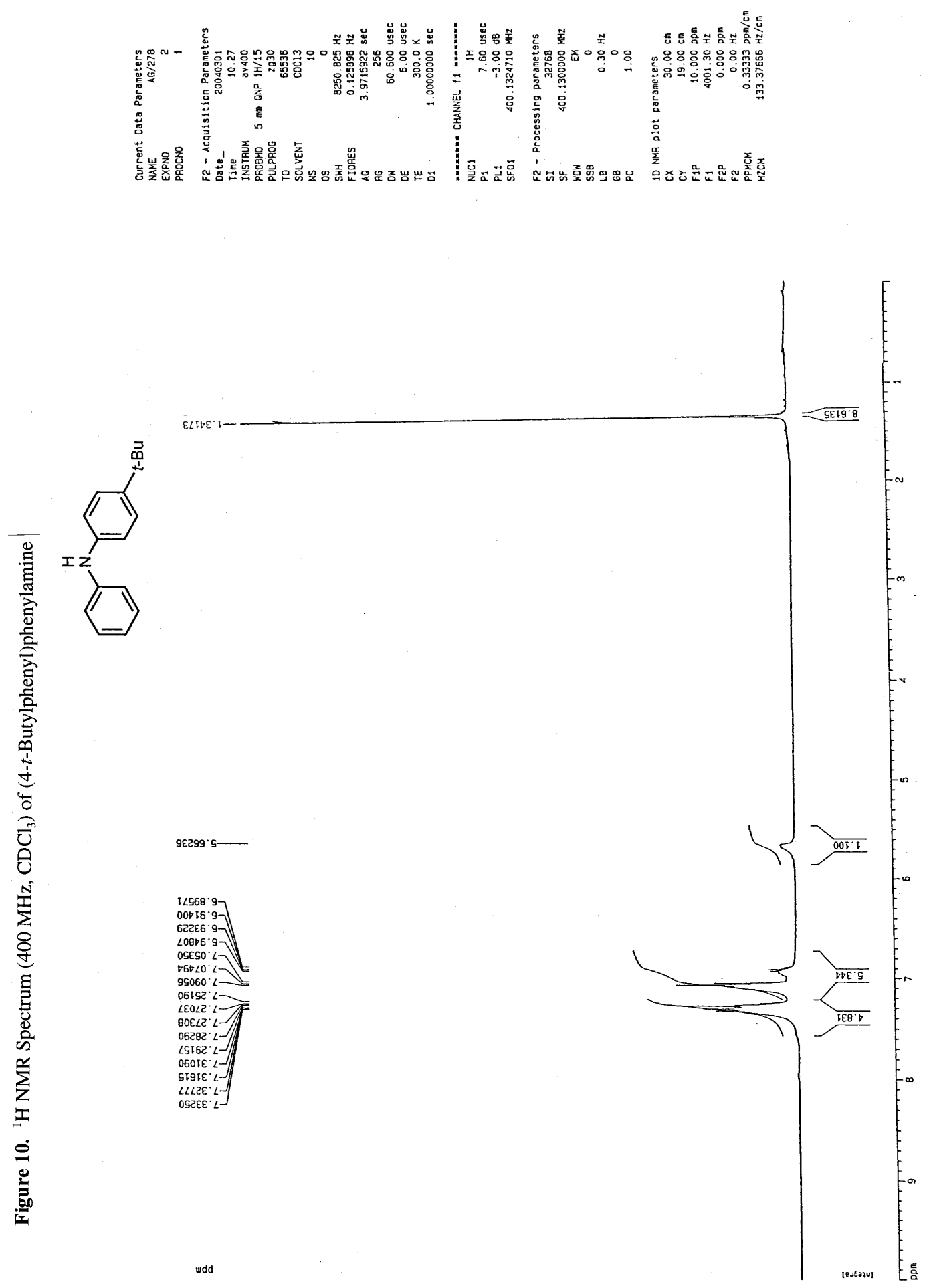

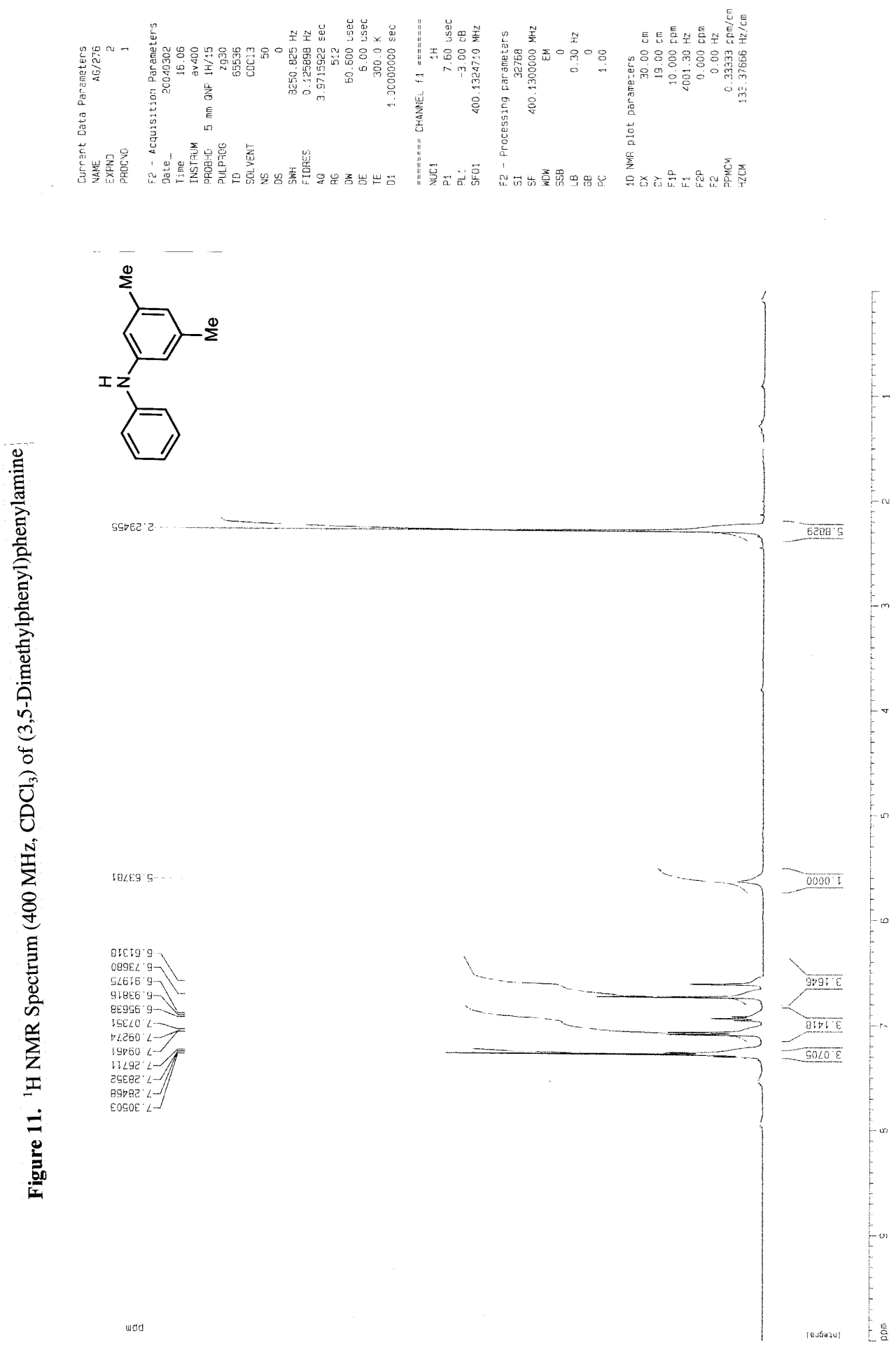

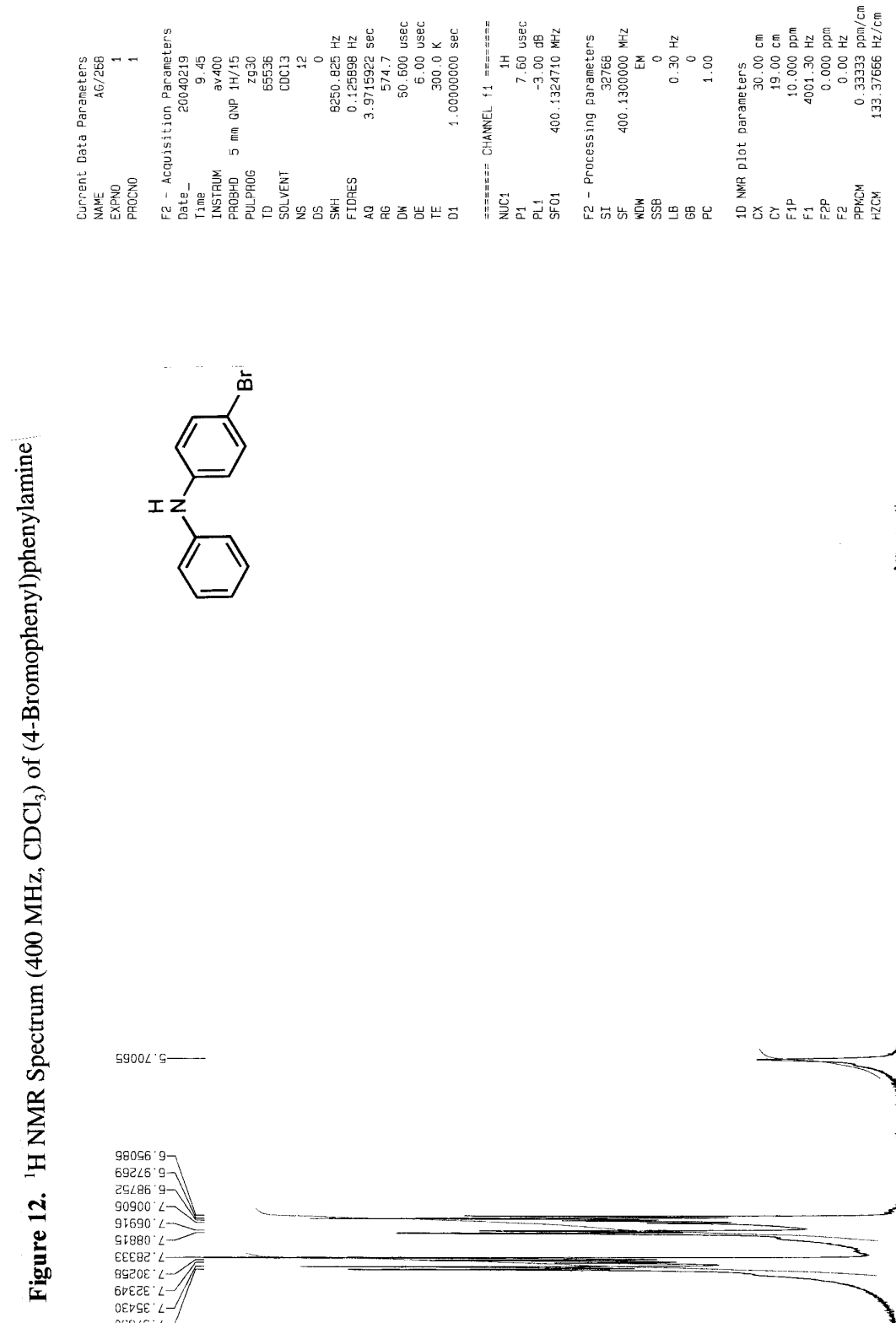

$5900<\mathrm{G}$

$\sum_{\text {Z }}^{Z}$

$98056.9-$
$592 \angle 5^{9}$
$2-1$

लi खद $2866^{\circ}$

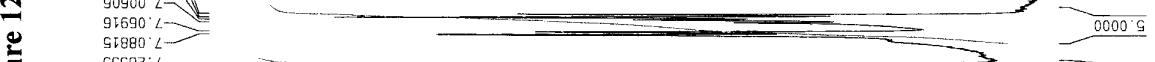

한

G5880 :-

EEEge : 2

BSEOE: $L$

OEV $9 / E^{\circ} L-$

wdo

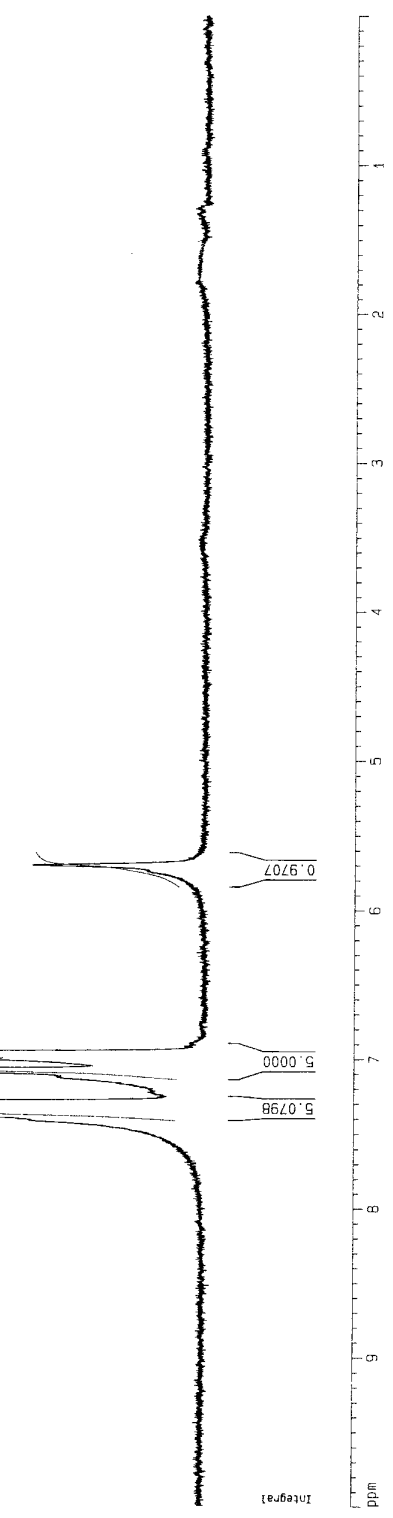



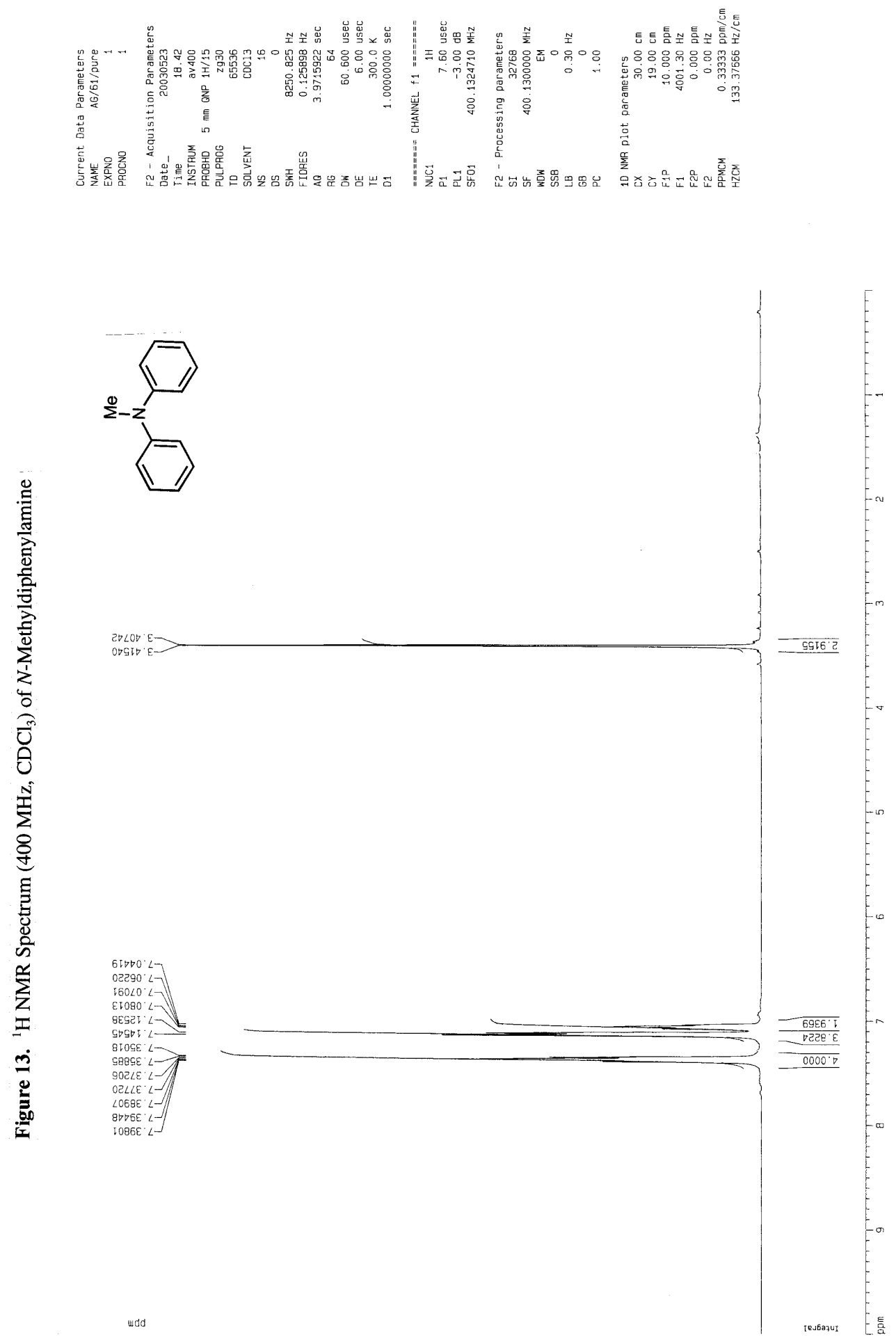

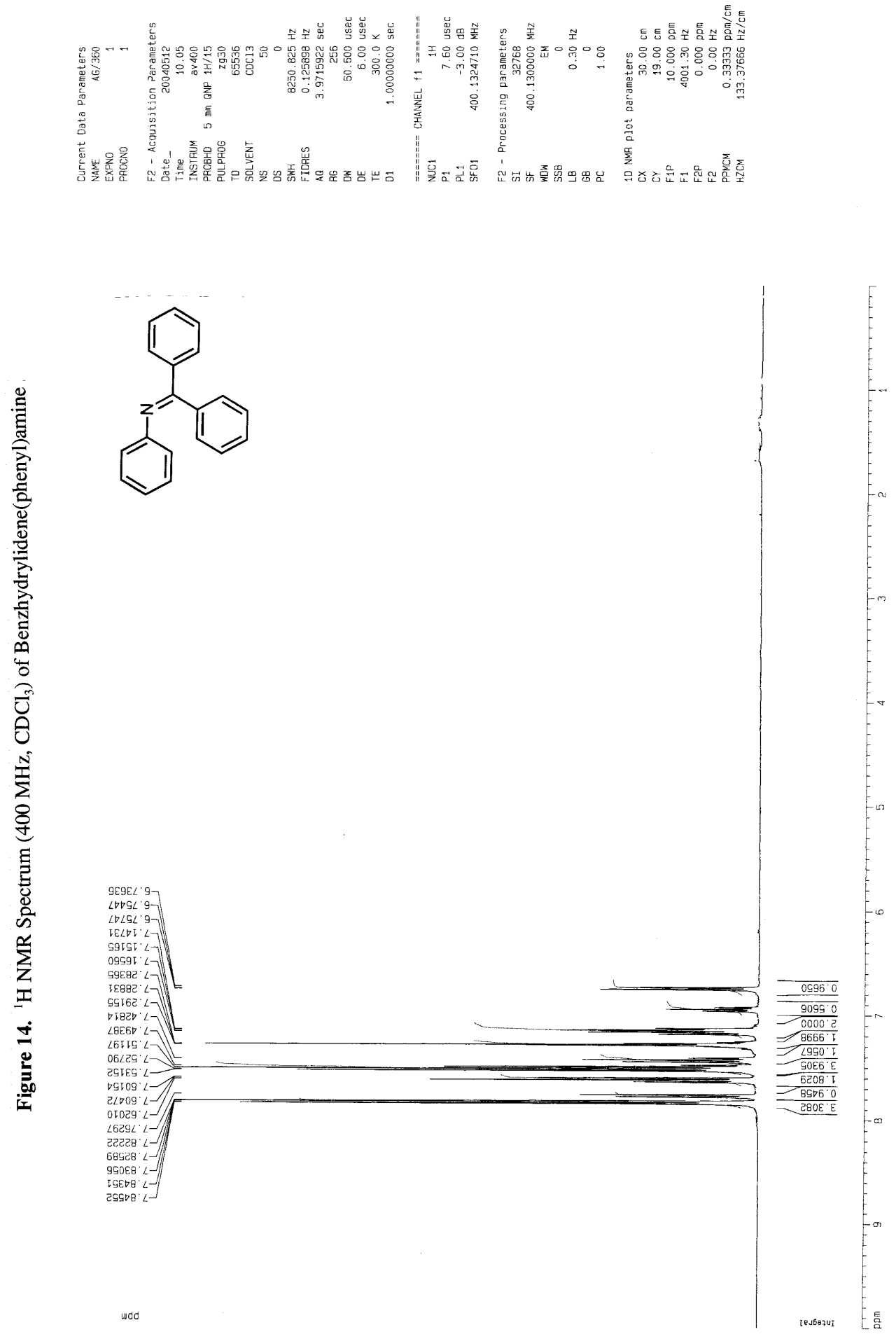

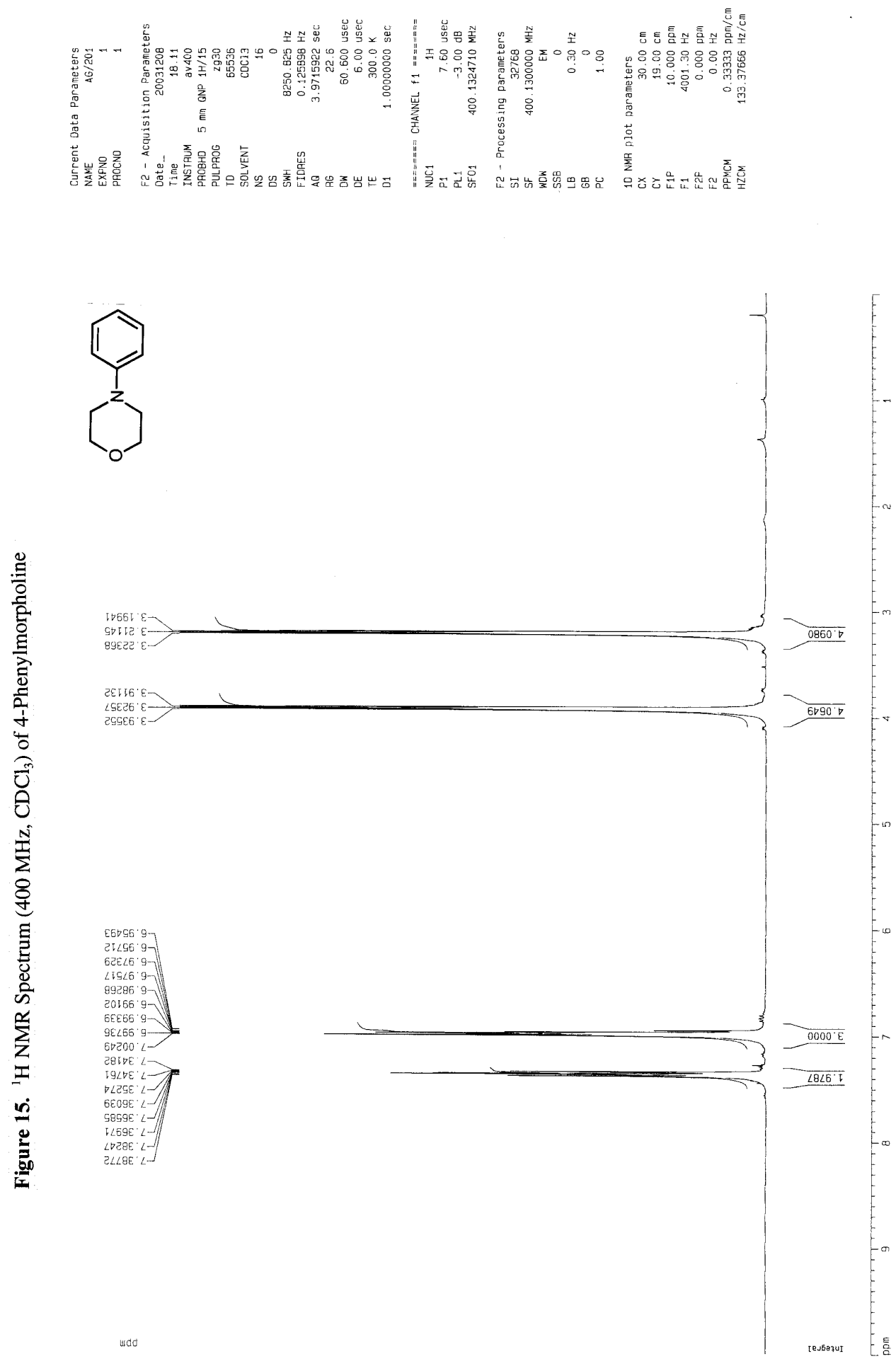


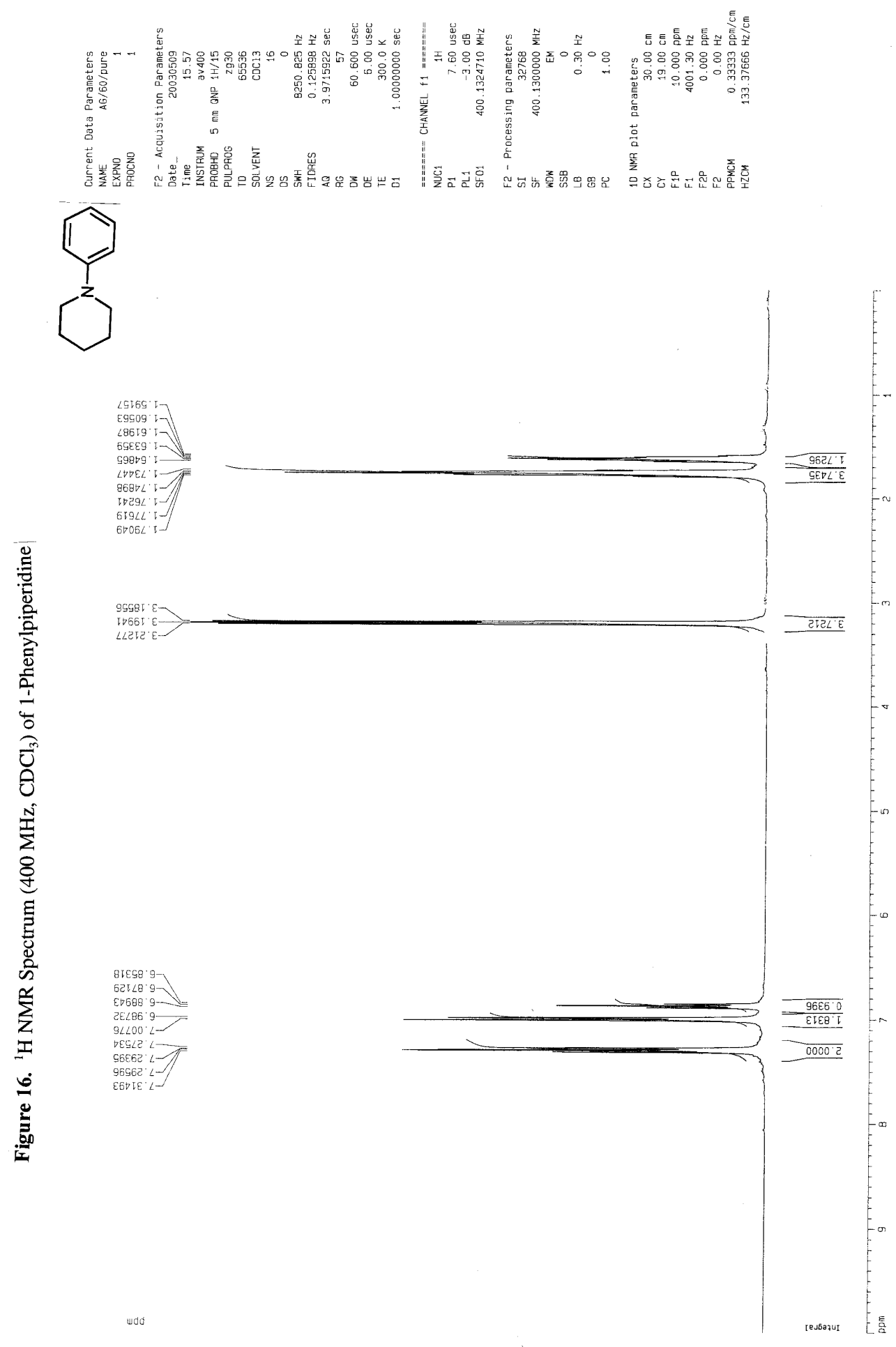




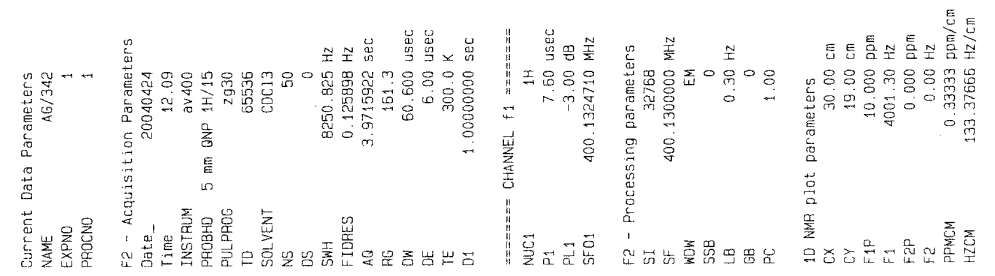<smiles>[C+]c1ccc([C]2CCCCC2)cc1</smiles>
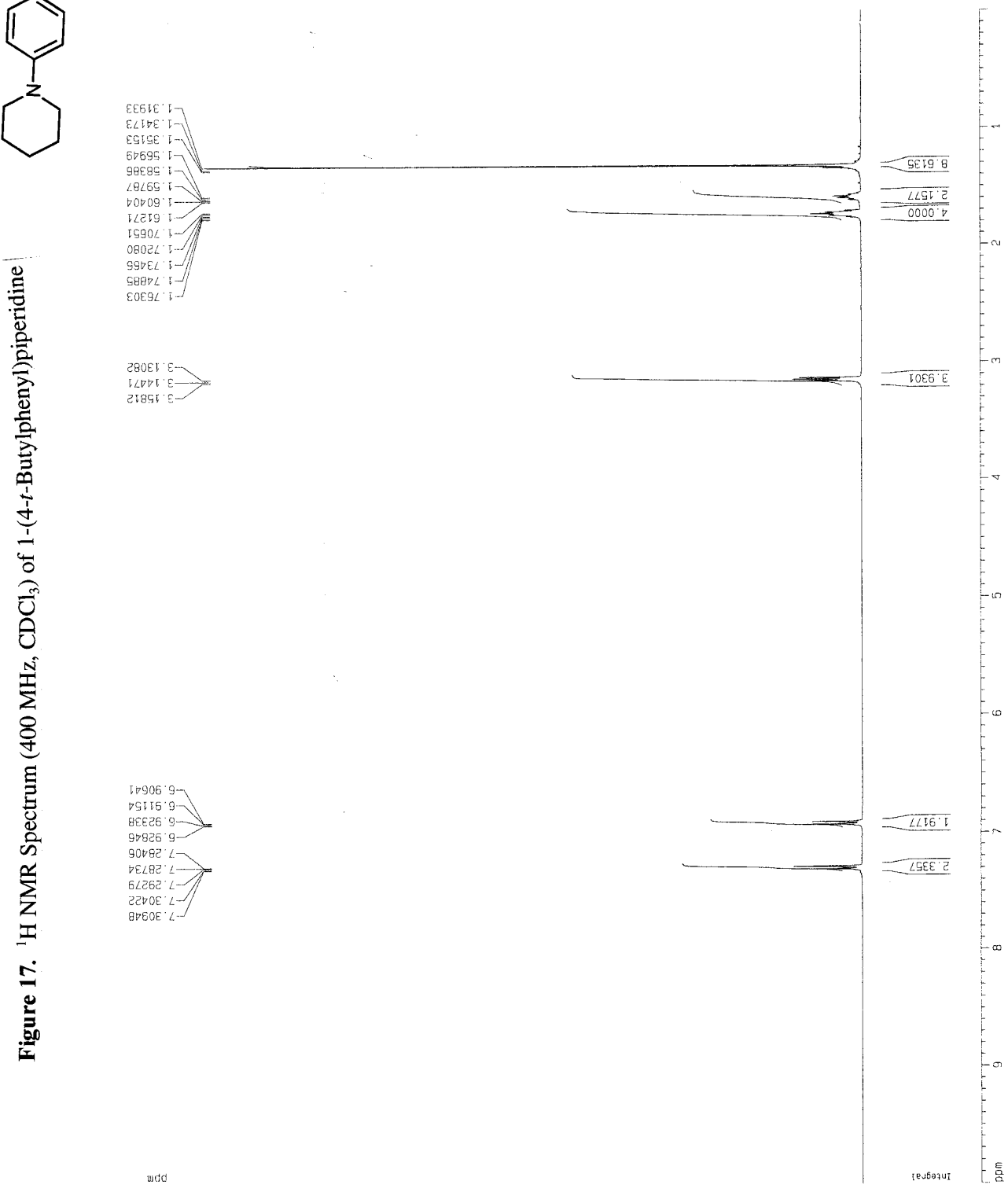

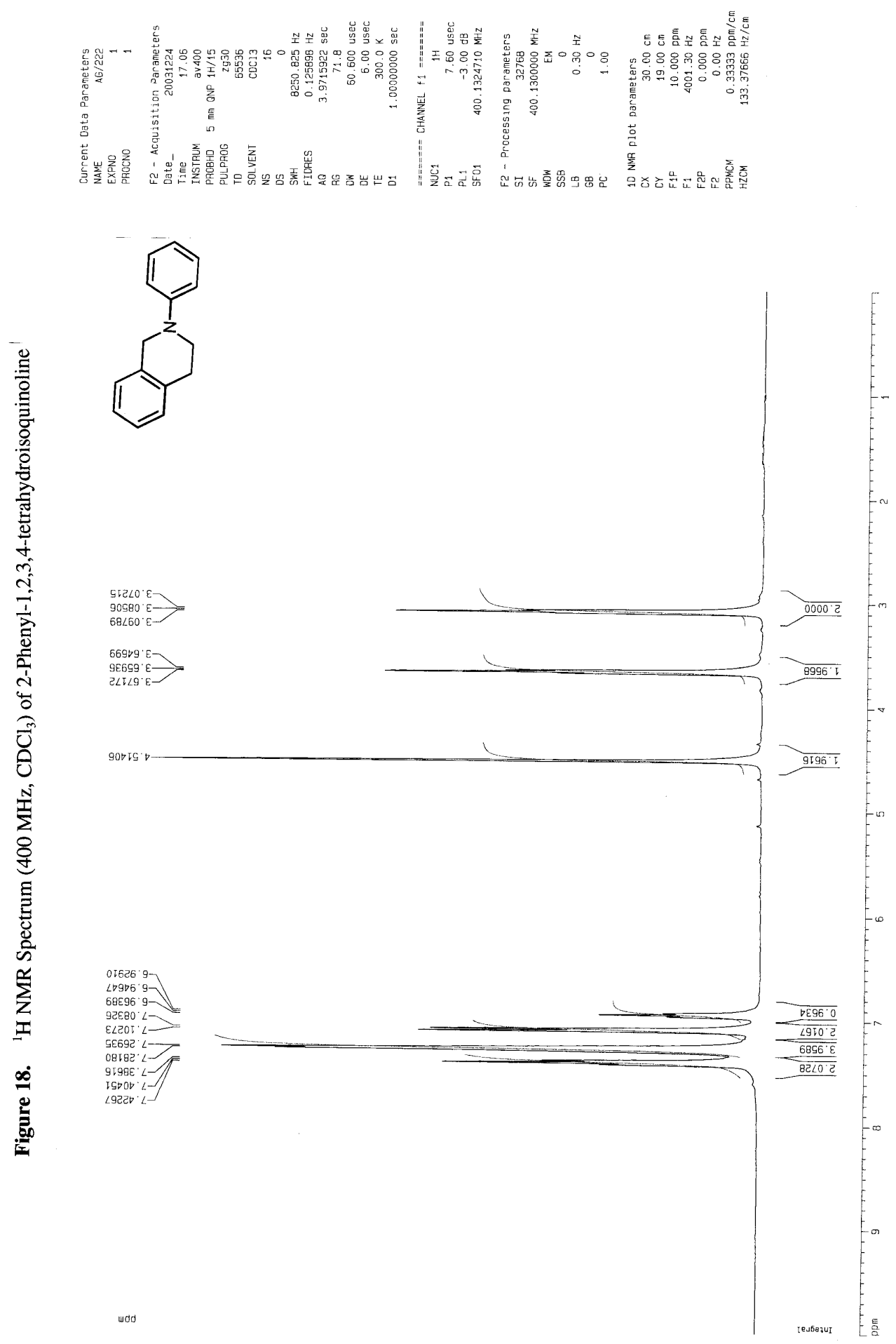
A Proposed Catalytic Cycle for Amination Reaction

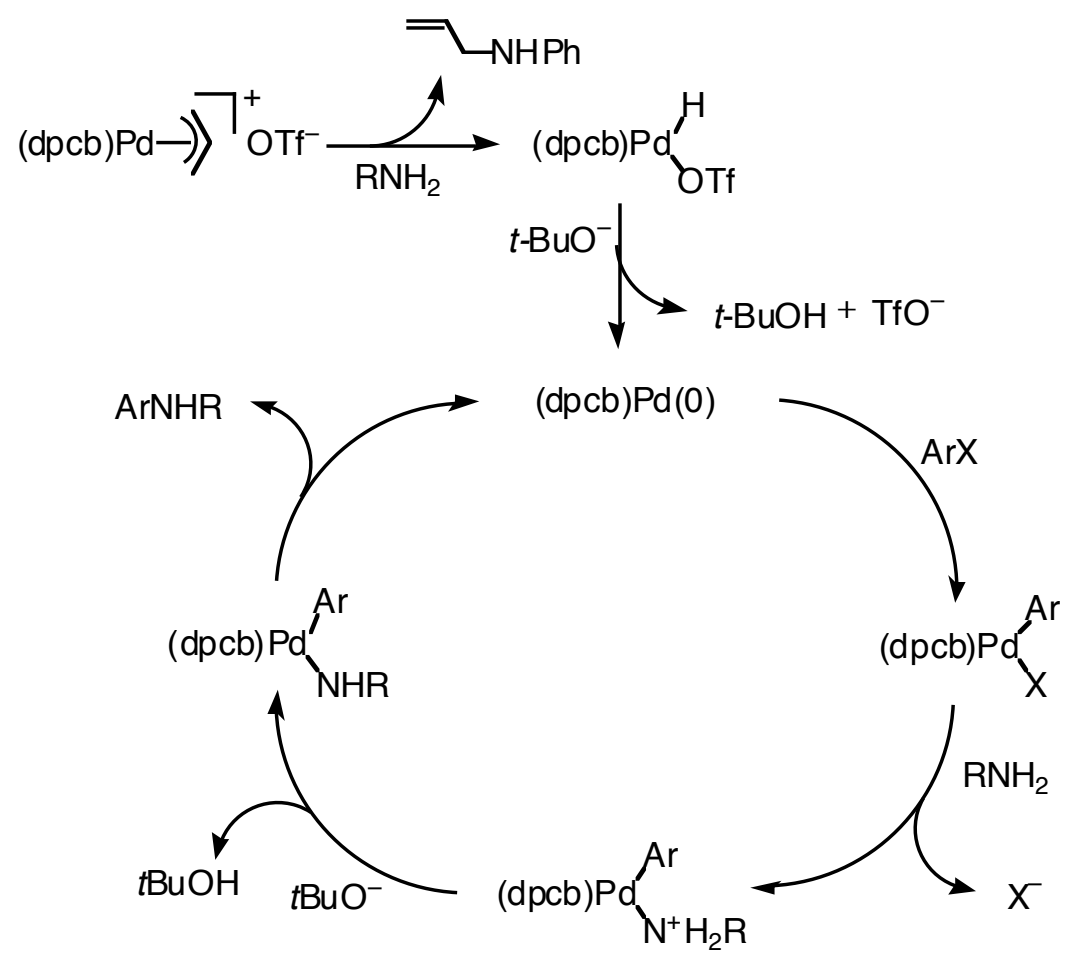




\section{References}

1. Ma, D.; Cai, Q.; Zhang, H. Org. Lett. 2003, 5, 2453.

2. Beller, M; Thiel, O. R.; Trauthwein, H.; Hartung, C. G. Chem. Eur. J. 2000, 6, 2513.

3. Kuwano, R.; Kondo, Y.; Matsuyama, Y. J. Am. Chem. Soc. 2003,125, 12104.

4. Quach, T. D.; Batey, R. A. Org. Lett. 2003, 5, 4397.

5. Fichert, T.; Massing, U. Tetrahedron Lett. 1998, 39, 5017

6. Kapp, F.; Sapountzis, L.; Knochel, P. Synlett. 2003, 885.

7. Driver, M. S.; Hartwig, J. F. J. Am. Chem. Soc. 1996,118, 7217.

8. Anderson, K. W.; Mendez-Perez, M.; Priego, J.; Buchwald, S. L. J. Org. Chem. 2003, 68, 9563.

9. Kang, S-K.; Lee, S-H.; Lee, D. Synlett. 2000, 1022.

10. Liu, Z.; Larock, R. C. Org. Lett. 2003, 5, 4673.

11. Samec, J. S. M.; Bäckvell, J-E. Chem. Eur. J. 2002, 8, 2955.

12. Xu, G.; Wang, Y-G. Org. Lett. 2004, 6, 985. 